\title{
Review of: "Investigating Carboxysome Morphology Dynamics with a Rotationally Invariant Variational Autoencoder"
}

\author{
Benedict Long ${ }^{1}$ \\ 1 Australian National University
}

Potential competing interests: The author(s) declared that no potential competing interests exist.

This interesting analysis utilizes a combination of chimeric fluorescent protein tagging, degron proteolysis of a target protein and epifluorescence image manipulation to provide a novel approach to the re-ordering of cyanobacterial carboxysomes resulting from shell-protein degradation. Carboxysomes are critically important, protein-bound, $\mathrm{CO}_{2}$-fixation organelles found in cyanobacteria and some proteobacteria, and are responsible for a significant portion of global $\mathrm{CO}_{2}$ sequestration into the biosphere [1]. They are extremely large, megaDalton-sized, icosahedral structures housing the enzyme Rubisco along with a specialized carbonic anhydrase enzyme and encapsulated in a protein shell. The biological construction and processing of carboxysomes is of broad research interest [2-5]. Recently, tools have been developed which enable the tracking and analysis of carboxysomes as they form and degrade $[2,6,7]$, providing novel insights into their biogenesis and breakdown.

The results presented in this preprint offer a new angle to assessing carboxysome re-processing upon proteolysis of the carboxysome shell protein, $\mathrm{CcmO}$, which the authors achieve utilizing their previously described targeted method which allows induction-driven expression of a specific protease which targets a modified form of CcmO in this case [7]. Combined with mNeonGreen fluorescent tagging of the carboxysomal cargo protein RbcS (the small subunit of Rubisco), this enables the tracking of the carboxysome contents during remodelling as the complex structure unravels.

This preprint primarily describes the rotationally invariant variational autoencoder (rVAE) system utilized to process fluorescence microscope images of cyanobacteria containing mNeonGreen-labelled RbcS protein. A broader description of this method in simpler terms would have been helpful for this reader, and reference to previous work by Kalinin, Zhang [8] is a good primer to enable those not familiar with image processing analysis. Understanding the biochemistry that underlies the observations is somewhat obscured by descriptions of the encoding/decoding utilized and I wonder if, in its current form, the preprint might miss the opportunity to inform both image analysis experts and those with a deep interest in carboxysomes.

The experimental approach appears sound and, based on previous work, is an exciting approach to asses 
changes in carboxysome morphology over time. However, I do wonder if there are checks and balances within the analysis to ensure that noise in the system can be accounted for (e.g. are the observed carboxysomes sufficiently small to ensure that all the mNeonGreen fluorescence is in the focal plane?) If this is not a problem and is described in previous work it would be good to know. The methodology used for microscopic analysis could be more detailed. I would also be very interested to know the resolution of the analysis.

While the study offers a novel approach to carboxysome morphology changes using the degron system, it could offer more to highlight its elaborations on image analysis approaches published previously (e.g. Kalinin, Zhang [8]) and perhaps more can be gleaned relating to carboxysome breakdown from the results to paint a picture of the observations made. For example, observations of carboxysomes 'collapsing' or moving to the poles of the cell could be discussed in terms of what we currently know of the role of CcmO [9] and the formation of Rubisco condensates driven by interactions with CcmM either inside an oxidized carboxysome or in the more reduced cytoplasm [3]. Are the observations specifically of carboxysomes or Rubisco-CcmM condensates (often described as polar bodies [9]), keeping in mind that it is an additional copy of RbcS which carries the fluorescent tag? The results are encouraging and I would enjoy seeing the interpretation fleshed out somewhat to see what can be uncovered and concluded regarding carboxysome morphology.

I short, this preprint offers significant promise in terms of the application of a very exciting means of controlled protein degradation, coupled with image analysis, to explore the morphological changes of carboxysomes over time. In its brevity it perhaps loses the impact of the new approach to image analysis and the opportunity to explore the outcomes in more detail. I'm eager to see this developed further.

1. Rae, B.D., et al., Functions, compositions, and evolution of the two types of carboxysomes: polyhedral microcompartments that facilitate $\mathrm{CO}_{2}$ fixation in cyanobacteria and some proteobacteria. Microbiology and Molecular Biology Reviews, 2013. 77(3): p. 357-379.

2. Hill, N.C., et al., Life cycle of a cyanobacterial carboxysome. Science Advances, 2020. 6(19): p. eaba1269.

3. Wang, H., et al., Rubisco condensate formation by CcmM in $\beta$-carboxysome biogenesis. Nature, 2019. 566(7742): p. 131-135.

4. Blikstad, C., et al., Discovery of a carbonic anhydrase-Rubisco supercomplex within the alphacarboxysome. bioRxiv, 2021: p. 2021.11.05.467472.

5. Chen, A.H., et al., The bacterial carbon-fixing organelle is formed by shell envelopment of preassembled cargo. PLoS One, 2013. 8(9): p. e76127.

6. Cameron, J.C., et al., Biogenesis of a bacterial organelle: the carboxysome assembly pathway. 
Cell, 2013. 155(5): p. 1131-1140.

7. Sakkos, J.K., et al., Orthogonal Degron System for Controlled Protein Degradation in Cyanobacteria. ACS Synthetic Biology, 2021. 10(7): p. 1667-1681.

8. Kalinin, S.V., et al., Disentangling Rotational Dynamics and Ordering Transitions in a System of Self-Organizing Protein Nanorods via Rotationally Invariant Latent Representations. ACS Nano, 2021. 15(4): p. 6471-6480.

9. Rae, B.D., et al., Structural determinants of the outer shell of b-carboxysomes in Synechococcus elongatus PCC 7942: roles for CcmK2, K3-K4, CcmO, and CcmL. PLoS One, 2012. 7(8): p. e43871. 